\section{Abstract}

\title{
Automated extraction of local defect resonance using the principal component analysis in lock-in ultrasonic vibrothermography
}

\author{
Seyed Ali Ghorashi ${ }^{\mathrm{a}}$, Farhang Honarvar ${ }^{\mathrm{a}}$, Morteza Tabatabaeipour ${ }^{\mathrm{b}, *}$ \\ ${ }^{a}$ NDE Lab, Faculty of Mechanical Engineering, K. N. Toosi University of Technology, 7 Pardis St., \\ Mollasadra Ave., Vanak Sq., Tehran, Iran \\ ${ }^{b}$ Centre for Ultrasonic Engineering, Department of Electronic and Electrical Engineering, University of \\ Strathclyde, Glasgow G11XW, UK
}

Ultrasonic vibrothermography is an emerging and promising nondestructive evaluation technique used for detection of surface and sub-surface defects. The heat-generating sources such as friction of the surface asperities of the defect and viscoelastic behavior of the structure may cause variations in non-linear elastic energy leading to the rise of temperature of the damaged area. In this paper, a Flat-Bottomed Hole (FBH) defect is modeled by finite element method in a polymethylmethacrylate (PMMA) structure. The desired information from this defect is retrieved by its local defect resonance (LDR) frequency which is estimated through a Principal Component Analysis (PCA). It is shown that the PCA algorithm can extract the LDR frequency of the FBH with high accuracy. The sample is then excited by a sine wave at its LDR frequency modulated by a low frequency corresponding to the thermal penetration depth. The lock-in amplitude and phase images are also generated at different modulation frequencies in order to find the optimal frequency in terms of contrast enhancement. The results of the finite element model are then verified by comparison with published experimental results and are found to be in very good agreement.

Keywords: Vibrothermography, local defect resonance, principal component analysis

\footnotetext{
${ }^{*}$ Corresponding author.

Email address: Morteza.Tabatabaeipour@strath.ac.uk (Morteza Tabatabaeipour)
} 


\section{Introduction}

Ultrasonic vibrothermography is a new nondestructive testing technique introduced in late 1970s [1]. High test productivity and adaptability to structures with complex geometries are some of the advantages of this new technique [1]. Recently, the introduction of the local defect resonance (LDR) has initiated considerable scientific research effort in ultrasonic vibrothermography $[2,3]$. It was also demonstrated that how the LDR, for a circular FBH defect and delamination, can be extracted by applying an image processing algorithm in every frequency bin to estimate the position and size of the defect [4]. However, this image processing algorithm may fail due to an assumption on the round shape of the LDR.

Results from earlier studies demonstrate that the heat generation induced by the LDR leads to high-temperature variation at the defective region $[3,5]$. In other words, an external mechanical energy source causes a temperature difference to be created between the defective and intact segments of the specimen. This temperature difference makes the defected region visible to an IR (infrared) camera [6]. The parameters that influence the generation of heat have been investigated by several researchers and it has been shown that three heating mechanisms of friction, plasticity, and viscoelasticity are the primary sources of heat generation [7]. These heat generation mechanisms may act individually or in combination. In the case of a flat-bottomed hole $(\mathrm{FBH})$ defect, the primary heat source is viscoelasticity, whereas in delamination and cracks, the combination of both viscoelasticity and friction plays the main role $[8,9]$. Furthermore, a direct relationship between the excitation frequency and the generated heat on the cracks has been reported by Holland et al. [10, 11].

Power dissipation at a defect is not the same as its surroundings. This temperature difference can be measured by using the lock-in principle for each pixel of the surface temperature [12]. In this way, the internal structure of the object can be evaluated by measuring the amplitudes and phase shifts of surface temperatures with respect to an input reference signal. The lock-in vibrothermography has been implemented for detection of a variety of defects including disbonding of ceramic coatings on metallic substrates [13] and a delamination/impact damages in the composite structures [14]. 
The objective of this paper is to evaluate the effectiveness of the Principal Component Analysis (PCA) algorithm in automatic selection of local defect resonances (LDRs). Furthermore, a search of the literature shows that what we currently know about this new technique is mostly based on empirical studies. It is rather difficult and expensive to obtain the optimal parameters by experiment. This paper provides the first numerical study for the entire process of the LDR-based lock-in vibrothermography. It also paves the way for further works on the optimization of this inspection process and defect characterization to be followed in the future.

In the first part of this paper, a ceramic piezo actuator mounted on a PMMA structure is modeled in the COMSOL Multiphysics finite element modeling software package. The piezo disk is then loaded with a chirp signal spanning a frequency range of 1-200 kHz to extract the local defect resonance of a flat-bottomed hole (FBH). The out-of-plane component of the signals is subsequently transformed to the frequency-domain and analyzed by using the PCA algorithm to extract the local defect resonance (LDR) of the FBH. The PCA results are then compared with the LDR frequency calculated by an eigenfrequency analysis which is a stand-alone technique.

In the second part of the paper, an amplitude modulated signal with the LDR frequency as a carrier and a low frequency as the message signal are fed into the ceramic piezo disk. Then, the lock-in thermography algorithm is applied to the acquired thermal waves to create the amplitude and phase images, and finally, the numerical results are compared with experimental results reported in $[2,3,15]$.

\subsection{Concept of local defect resonance}

If no structural degradation is caused by ultrasonic excitation, the amplitude of ultrasonic vibrations in a vibrothermography test can be increased to enhance the effectiveness of this technique. However, this is not always economical or doable due to hardware limitations. As an alternative approach, exciting the test piece with a Local Defect Resonance (LDR) frequency, which is the resonance frequency of the local defect, can pump the maximum amount of energy into the defect region. Therefore, it is good to know the LDR for any type 

effective mass and effective rigidity are as follows [16]:

$$
\begin{gathered}
m_{\text {eff }}=1.8 m, \quad m=\rho \pi R^{2} \\
k_{\text {eff }}=\frac{198 \pi D}{R^{2}} \sqrt{\frac{k_{\text {eff }}}{m_{\text {eff }}}}, \quad D=\frac{E d^{3}}{12\left(1-\nu^{2}\right)}
\end{gathered}
$$

87 where $D$ is the bending stiffness, $R$ is the radius of the circular defect, $d$ is the residual 97 specific heat capacity.

wall thickness of the FBH, $E$ is the modulus of elasticity of the specimen, $\nu$ is the Poisson's ratio, $\rho$ is the density and $m$ is the residual mass of the FBH. By combining equations (1), (2) and (3), the LDR frequency of a circular FBH is found to be $[4,16]$ :

$$
f_{L D R}=\frac{1}{2 \pi} \sqrt{\frac{k_{e f f}}{m_{e f f}}} \approx \frac{1.6 d}{R^{2}} \sqrt{\frac{E}{12 \rho\left(1-\nu^{2}\right)}}
$$

\subsection{Thermal analytical approach}

According to the FourierâĂŹs law for a transient heat transfer in a solid with an internal heat source, the temperature distribution is calculated from the following equation [18]:

$$
k_{x} \frac{\partial^{2} T}{\partial x^{2}}+k_{y} \frac{\partial^{2} T}{\partial y^{2}}+k_{z} \frac{\partial^{2} T}{\partial z^{2}}+q=\rho C_{p} \frac{\partial T}{\partial t}
$$

where $T(x, y, z, t)$ is the time-dependent temperature of point $(x, y, z), k(x, y, z, t)$ is the thermal conductivity which has identical values along all three coordinate axes due to the isotropic properties of the sample, $q$ is the volumetric heat generation in the sample and $C_{p}$ is the 
The boundary condition on the upper surface of the plate, considering convection heat transfer, is:

$$
-\hat{n} \cdot(-k \nabla T)=h\left(T_{s}-T\right)
$$

Where $h=5\left(\frac{\mathrm{W}}{\mathrm{m}^{2} \mathrm{~K}}\right)$ is the convective heat transfer coefficient.

The generated volumetric heat can be written as [19, 20]:

$$
q=\eta \omega W
$$

In equation (7), $\eta$ is the structural loss factor, $\omega\left(\frac{\mathrm{rad}}{\mathrm{s}}\right)$ is the angular frequency, $W\left(\frac{\mathrm{J}}{\mathrm{m}^{3}}\right)$ is the elastic strain energy density defined at a specific time as follows [19, 20]:

$$
W=\frac{E u_{z}^{2}}{2\left(1-\nu^{2}\right)}\left[\left(\frac{\partial^{2} U}{\partial x^{2}}\right)^{2}+\left(\frac{\partial^{2} U}{\partial y^{2}}\right)^{2}+2 \nu \frac{\partial^{4} U}{\partial x^{2} \partial y^{2}}+2(1-\nu)\left(\frac{\partial^{2} U}{\partial x \partial y}\right)^{2}\right]
$$

where $U=U(x, y, z, t)$ is the displacement which appears due to the harmonic excitation of the piezoelectric crystal and $u_{z}$ is the out-of-plane component of displacement at the surface the of plate.

By incorporating equations (7) and (8), the generated heat can be calculated from Eq (9) which shows that the induced heat is a quadratic function of the out-of-plane component of displacement, $u_{z}$ :

$$
q=\frac{\eta \omega E u_{z}^{2}}{2\left(1-\nu^{2}\right)}\left[\left(\frac{\partial^{2} U}{\partial x^{2}}\right)^{2}+\left(\frac{\partial^{2} U}{\partial y^{2}}\right)^{2}+2 \nu \frac{\partial^{4} U}{\partial x^{2} \partial y^{2}}+2(1-\nu)\left(\frac{\partial^{2} U}{\partial x \partial y}\right)^{2}\right]
$$

\section{Simulation model setup}

In order to evaluate the LDR-based vibrothermography, a PMMA sample is modelled in COMSOL Multiphysics finite element modeling software package with dimensions of $200 \times$ $40 \times 3 \mathrm{~mm}^{3}$, containing an FBH defect with a radius of $10 \mathrm{~mm}$ and a depth of $2 \mathrm{~mm}$ as shown in Fig. 1. The material properties of the sample are given in Table 1. Three different studies have been conducted in COMSOL Multiphysics for the analysis of the model as follows:

- Eigenfrequency analysis: To find the local defect resonance frequency. This is a standalone analysis, meaning that there is no need for any signal analysis to calculate the LDR frequency. The piezo actuator can be disabled during this analysis. 
- Time-domain analysis: To monitor the signals generated by a piezo actuator from the defect region for Principal Component Analysis (PCA). More details on the wave propagation and the piezo actuator are given in Section 2.1.

- Frequency domain analysis coupled with a time-domain study of the heat transfer: To evaluate the thermal waves generated by a wave-modulated signal in order to plot the thermosonic images. The frequency-domain study allows us to capture long duration of signals for numerical analysis. Furthermore, the structural loss factor can only be applied in the frequency domain analysis module of the software.

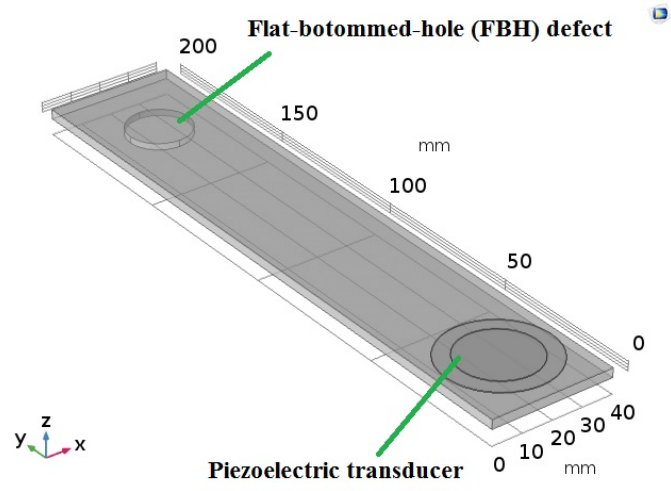

Figure 1: Geometry of the simulated PMMA model, showing the position of both an FBH defect and a piezoelectric actuator.

\subsection{Simulation of wave propagation and the piezo actuator}

To find the LDR frequency, we use an excitation frequency range of 1-200 kHz. This range of frequencies can generate several Lamb wave modes in the plate-like structure. The suitable mesh size is found from the dispersion curves plotted by using the GUIGUW software [21]. The maximum group velocity in this range is approximately $2158\left(\frac{\mathrm{m}}{\mathrm{s}}\right)$ and the minimum wavelength is $5.6 \mathrm{~mm}$. To capture the dynamics of ultrasonic vibrations, at least 8 spatial grid points are taken. Furthermore, the Courant-Friedrichs-Lewy (CFL) stability criterion gives the following strict requirement relationship between the size of elements $\Delta x$ 
Table 1: Material properties of the PMMA plate in the FEM analysis

\begin{tabular}{ll}
\hline Parameter & Value \\
\hline Young modulus $(E)$ & $6.18 \mathrm{GPa}$ \\
Poisson ratio $(\nu)$ & 0.3216 \\
Denity $(\rho)$ & $1190\left(\frac{\mathrm{k} g}{\mathrm{~m}^{3}}\right)$ \\
Thermal conductivity $(k)$ & $0.1905\left(\frac{\mathrm{W}}{\mathrm{m}^{2} \mathrm{~K}}\right)$ \\
Specific heat capacity $\left(C_{p}\right)$ & $1440\left(\frac{\mathrm{J}}{\mathrm{kg}{ }^{\circ} \mathrm{C}}\right)$ \\
Thermal diffusivity coefficent $\left(\alpha=\frac{k}{\rho C_{p}}\right)$ & $1.11 \times 10^{-7}\left(\frac{\mathrm{m}}{\mathrm{s}}\right)$ \\
Structural damping coefficient $(\beta)$ & 0.02 \\
Initial temperature $\left(T_{0}\right)$ & $20{ }^{\circ} \mathrm{C}$
\end{tabular}

and the time-step size of the solver $\Delta t$ :

$$
\Delta t \leq \frac{\Delta x}{\sqrt{3} C_{\max }}
$$

136

where $C_{\text {max }}$ is the maximum speed of the ultrasonic waves [22, 23]. A time-step of $1 \times 10^{-7}(\mathrm{~s})$ is found to be suitable for this model and a tetrahedral mesh was applied to the structure.

To verify the model by the experimental data available in the literature $[2,15]$, the identical piezo-ceramic actuator (Conrad Electronic $\mathrm{GmbH}$ ) was used to excite the specimen in the simulation $[2,24]$. As shown in Fig. 2, several resonance peaks are observed in the frequency range of $1-200 \mathrm{kHz}$, which depend on the piezo actuator dimensions and its material properties. Note that the local defect resonance (LDR) is not associated with any of these piezo resonances. In other words, the resonances of the piezoceramic do not control the local defect resonance. The specifications of this piezo actuator are listed in Table 2 . The out-of-plane signal components from the upper surface $(z=3 \mathrm{~mm})$ of the defect region are monitored for further analysis. The spatial step of $1 \mathrm{~mm}$ was chosen for monitoring the signals in both $x$ and $y$ directions. 

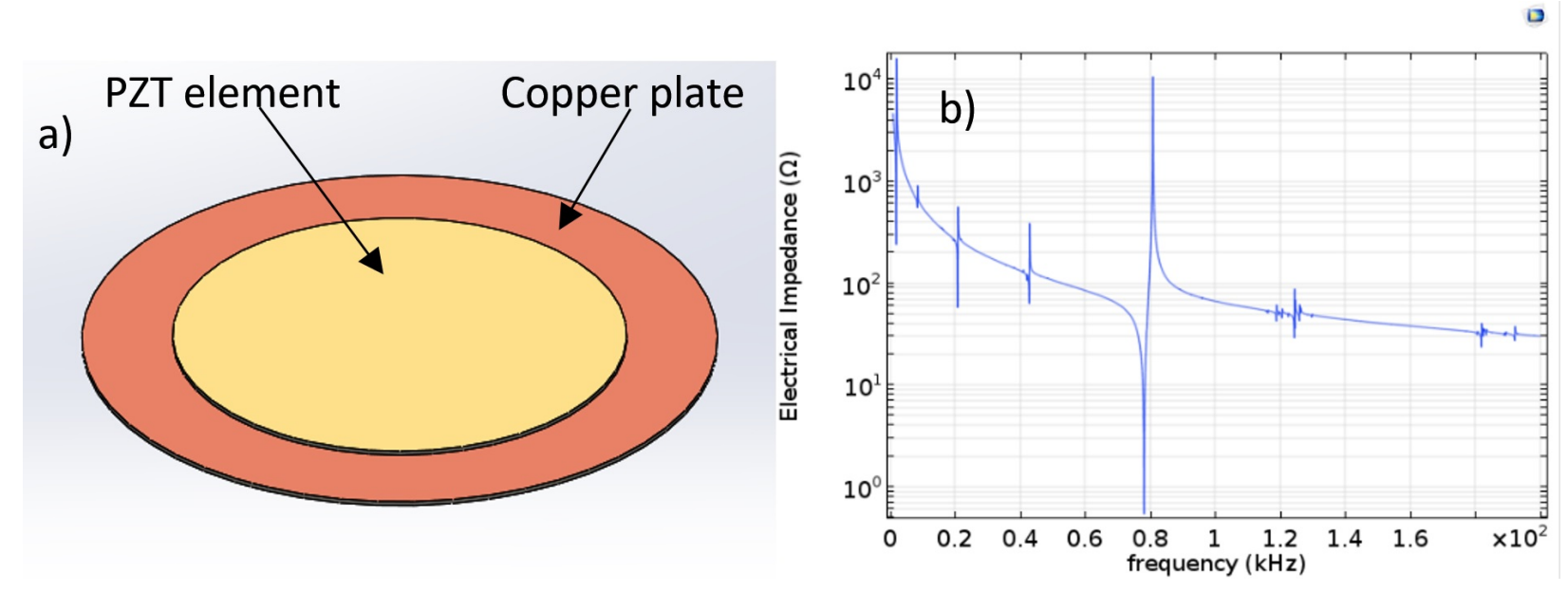

Figure 2: a) The piezo actuator model used in simulations, b) Impedance analysis of the simulated piezoelectric actuator.

Table 2: Piezo actuator specifications exploited in the simulation.

\begin{tabular}{ccccc}
\hline $\begin{array}{c}\text { Element } \\
\text { diameter }(\mathrm{mm})\end{array}$ & $\begin{array}{c}\text { Plate diameter } \\
(\mathrm{mm})\end{array}$ & $\begin{array}{c}\text { Element } \\
\text { thickness }(\mathrm{mm})\end{array}$ & $\begin{array}{c}\text { Plate thickness } \\
(\mathrm{mm})\end{array}$ & Plate material \\
\hline 25 & 35 & 0.26 & 0.51 & Copper \\
\hline
\end{tabular}

\section{Estimation of Local Defect Resonance (LDR)}

\subsection{Eigenfrequency analysis}

The LDR of an FBH defect is calculated by using three different approaches which are: 1) a theoretical equation, 2) eigenfrequency analysis, and 3) Principal Component Analysis (PCA). Eigenfrequency analysis is a stand-alone process and for this analysis, we do not need to measure any signals for estimating the LDR. Therefore, the LDR obtained from eigenfrequency analysis is used as the benchmark.

The eigenfrequency analysis does not only provide the local defect resonance but it also illustrates the mode-shape. Figure 3 shows the results of eigenfrequency analysis carried out in COMSOL Multiphysics at two different frequencies. In Fig. 3a, the excitation frequency coincides with one of the resonance frequencies of the whole sample and therefore, the sample is seen to be vibrating at its corresponding resonance mode. In Fig. 3b, the excitation 

the defect region is much higher than the rest of the test sample.
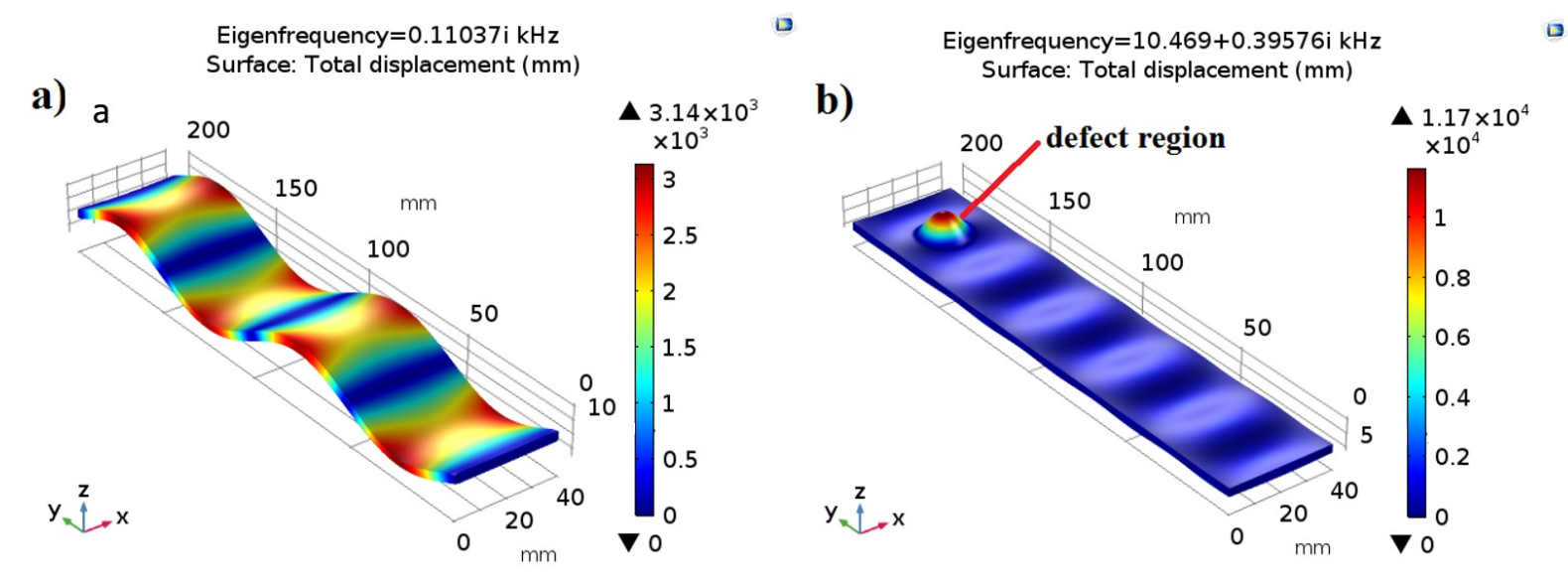

Figure 3: Eigenfrequency analysis performed by using COMSOL Multiphysics at two different frequencies, a) an arbitrary frequency showing one of the resonance modes of the sample, b) at LDR of the FBH defect where large displacements at the defect zone are observed.

\subsection{Principal component analysis}

Principal component analysis (PCA) is a useful technique for analyzing a large dataset by increasing its interpretability while minimizing information loss [25, 26]. PCA can be described as an N-dimensional ellipsoid matched to the data so that each axis of this ellipsoid declares a principal component [26]. The large axes of this ellipsoid, which correspond to the maximum variances of the variables, are retained and the small axes with low variances are removed. Since LDR produces maximum displacement on top of the defect, it can be a powerful tool for extracting the desired information from a $2 \mathrm{D}$ array of signals that are measured on top of the defect.

The out-of-plane displacement components are measured on a $31 \times 31 \mathrm{~mm}^{2}$ region of interest on top of the defect region. This gives a data set of $10000(\mathrm{n}) \times 961(\mathrm{p})$, where $\mathrm{n}$ and $\mathrm{p}$ represent the number of samples in each measurement and the total number of measurements, respectively. This 2D array of time-domain signals is then transformed into frequency-domain, resulting in a matrix with similar dimensions. The rows of this matrix 
correspond to the FFT magnitudes at different frequency bins and the columns correspond to different measurements made on the region of interest. The PCA algorithm is then applied to this matrix to extract the LDR frequency. In other words, after setting the distribution of the data around the origin, the data array is decomposed into three matrices utilizing a singular value decomposition (SVD) analysis [25, 26]. Next, by sorting the eigenvalues, an eigenvector corresponding to the first or maximum eigenvalue can be described as an axis that the most interpretability of data is visible. Figure 4 illustrates the magnitudes of the eigenvalues in a descending order which quickly tends to zero. This trend is consistent with PCA images as shown in Fig. 5.

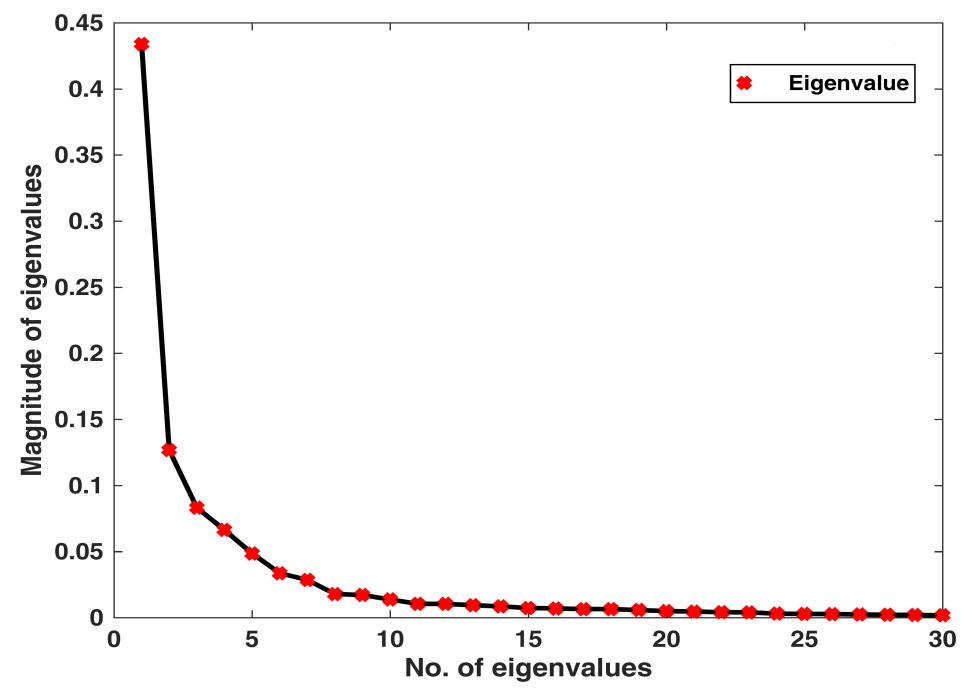

Figure 4: The magnitude of eigenvalues in a descending order showing quick convergence to zero.

Figure 6 shows the projection of data onto the first, second, third and fourth axes. Each point of this plot represents a frequency bin, allowing us to extract the LDR frequency by finding the higher values of the principal components. By extracting the matrix of features around the second principal component, the LDR frequency of the FBH is found to be 10.4 $\mathrm{kHz}$. It is important to note that the LDR frequency of the FBH defect can only be depicted (without any other distracting frequency components) on the second principal eigenvalue (see Fig. 6). In other words, the image obtained from the second principal component only contains the LDR frequency of the FBH. The matrix of features extracted from the first 

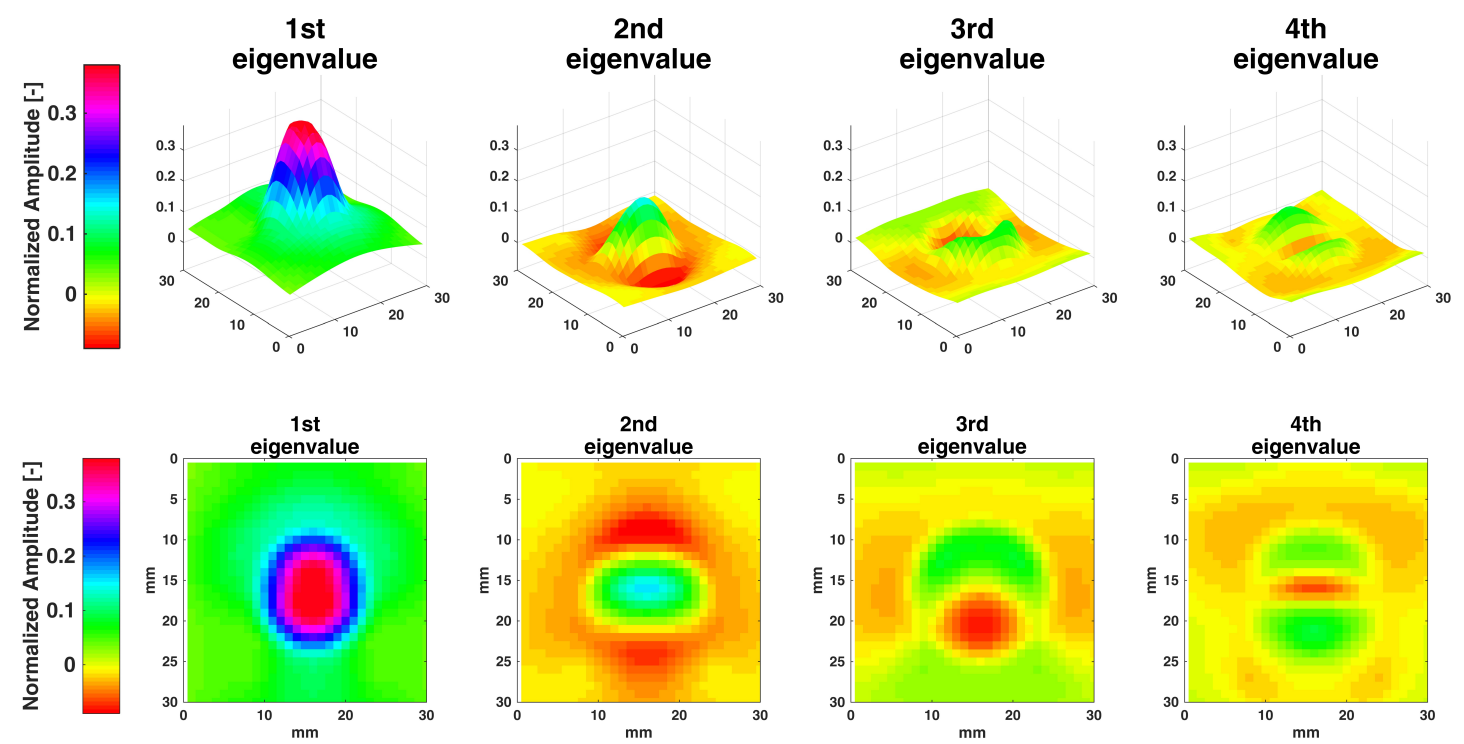

Figure 5: PCA images of different modes showing the consistency of the eigenvectors with the results reported in Fig. 4.

principal eigenvalue (see Figs. 5 and 6) includes additional frequencies in addition to the LDR frequency, which are due to the resonance frequencies of the sample (e.g. at $3.5 \mathrm{kHz}$ ) and the higher harmonics of the FBH defect (e.g. at $17.8 \mathrm{kHz}$ ).

\subsection{A comparison between local defect resonances}

In this section, the calculated and the experimental LDR frequencies reported in $[2,15]$ are compared with the calculated LDR frequency through an eigenfrequency analysis. The relative error is calculated by equation (11) and is repotrted in Table 4.

$$
\text { error }=\frac{\left|f_{L D R, \text { eigenfrequency }}-f_{L D R, i}\right|}{f_{L D R, \text { eigenfrequency }}} \quad i=1,2,3
$$

where $f_{L D R, i}, i=1,2,3$ are the LDR frequencies obtained by theory, PCA algorithm and experiment, respectively. Note that the experimental LDR frequency given in $[2,15]$ is 11 kHz. This comparison confirms that PCA analysis of the monitored signals can extract the local defect resonance (LDR) frequency with little error. 


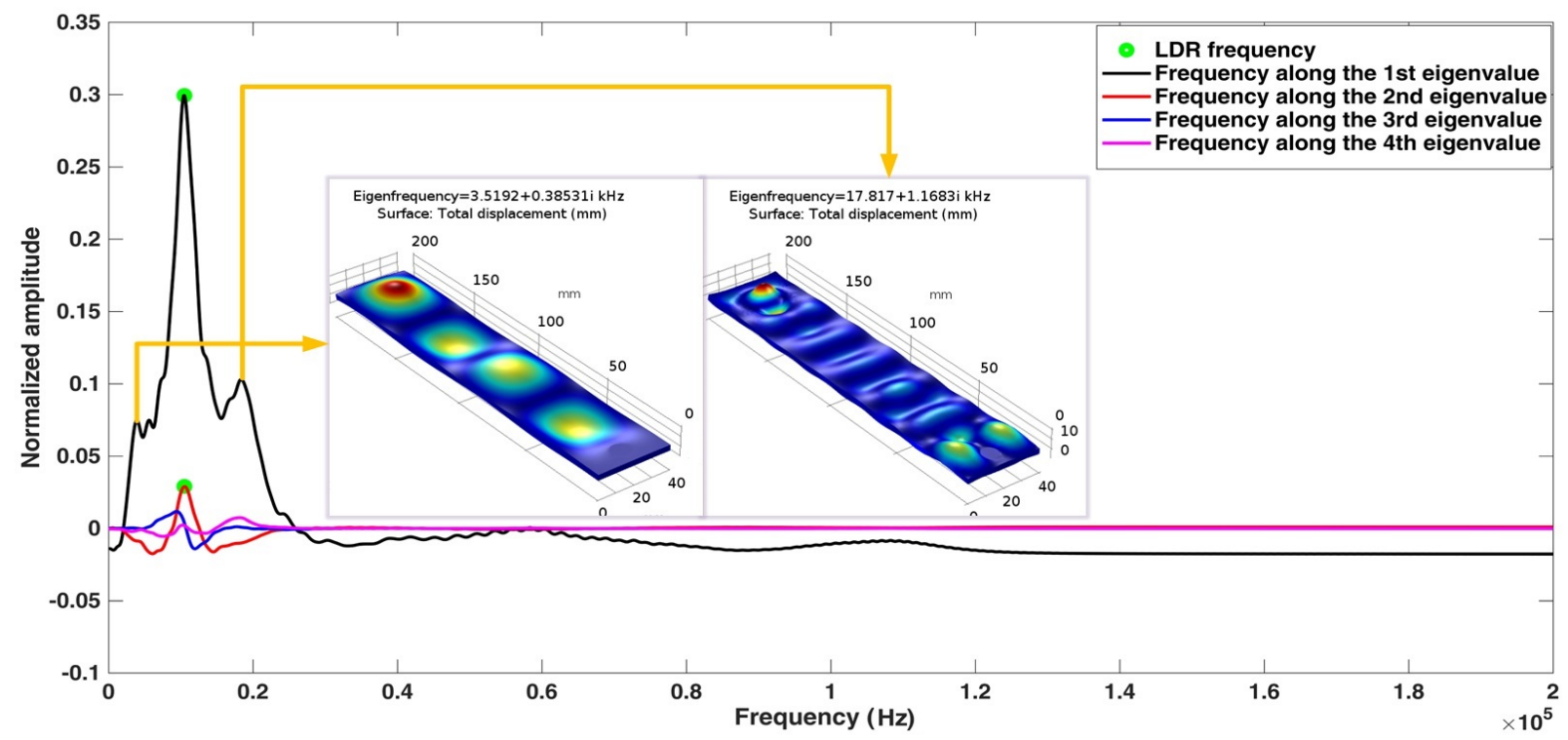

Figure 6: A comparison between the first, second, third and fourth principal axes which shows that only the 1st and 2nd principal components depict the LDR frequency of the FBH defect at $10.4 \mathrm{kHz}$. Note that the 2nd principal component has a lower amplitude compared to the 1st mode. In contrast, the 2nd mode only provides the LDR peak whereas the 1st mode gives rise to multiple local peaks corresponding to higher resonance frequencies of FBH defect and/or the sample resonances in addition to LDR frequency.

Table 3: Comparison of LDR frequencies obtained from theory, PCA algorithm and experiment.

\begin{tabular}{cccc} 
Method & Source & LDR frequency $[\mathrm{kHz}]$ & Error [\%] \\
\hline Theoretical equations & Eq. 4 & 11.12 & 6.21 \\
PCA algorithm & Fig. 6 & 10.4 & 0.67 \\
Experimental analysis & {$[2,15]$} & 11 & 5.06
\end{tabular}

\section{Thermal patterns via lock-in algorithm}

\subsection{Thermal penetration depth}

To investigate the thermal images, the sample is excited by a sinusoidal frequency which combines a modulated carrier signal $U_{c}(t)$ :

$$
U_{c}(t)=u_{1} \sin \left(2 \pi f_{1} t+\phi_{1}\right)
$$


with a message signal $U_{m}(t)$;

$$
U_{m}=u_{2} \sin \left(2 \pi f_{2} t+\phi_{2}\right)
$$

$$
\frac{\partial^{2} T}{\partial x^{2}}+\frac{\partial^{2} T}{\partial y^{2}}+\frac{\partial^{2} T}{\partial z^{2}}+\frac{q}{k}=\frac{1}{\alpha} \frac{\partial T}{\partial t}
$$

Table 4: Theoretically calculated thermal diffusion length and the blind frequency for the FBH defect. Amplitude Image
Phase Image where $C$ is a correlation constant and $\alpha$ is the thermal diffusivity. The values of $C=1$ and $1.5<C<2$ are usually used for calculation of amplitude and phase, respectively [27, 28]. These images can be constructed by a lock-in algorithm which will be discussed in more detail in section 4.2. Table 4 presents the calculated diffusion lengths and the blind frequencies for both amplitude and phase images based on the depth of the defect.

There is no sufficient phase contrast for defect detection at frequencies higher than the limiting frequency $f_{2}$, also known as the blind frequency [27]. By substituting Eq (17) into Eq (5), the thermal distribution pattern will turn out to be:

where $u_{1}$ and $u_{2}$ are the amplitudes of two sine signals. The LDR frequency, $f_{1}$, is carried by a lower frequency signal, $f_{2}$, which is related to the thermal diffusion length $\mu$. This thermal diffusion length, $\mu$, is a decay rate of thermal waves which is correlated to the depth of the defect, $Z$, as follows [27, 28]:

$$
Z=C \sqrt{\frac{\alpha}{\pi f_{2}}}=C \mu, \alpha=\frac{k}{\rho C_{p}}
$$

\begin{tabular}{cccc}
\hline \multicolumn{2}{c}{ Amplitude Image } & \multicolumn{2}{c}{ Phase Image } \\
\hline Diffusion length $(\mathrm{mm})$ & Frequency $(\mathrm{Hz})$ & Diffusion length $(\mathrm{mm})$ & Frequency $(\mathrm{Hz})$ \\
2 & 0.009 & 1.11 & 0.03
\end{tabular}



wave acquisition.

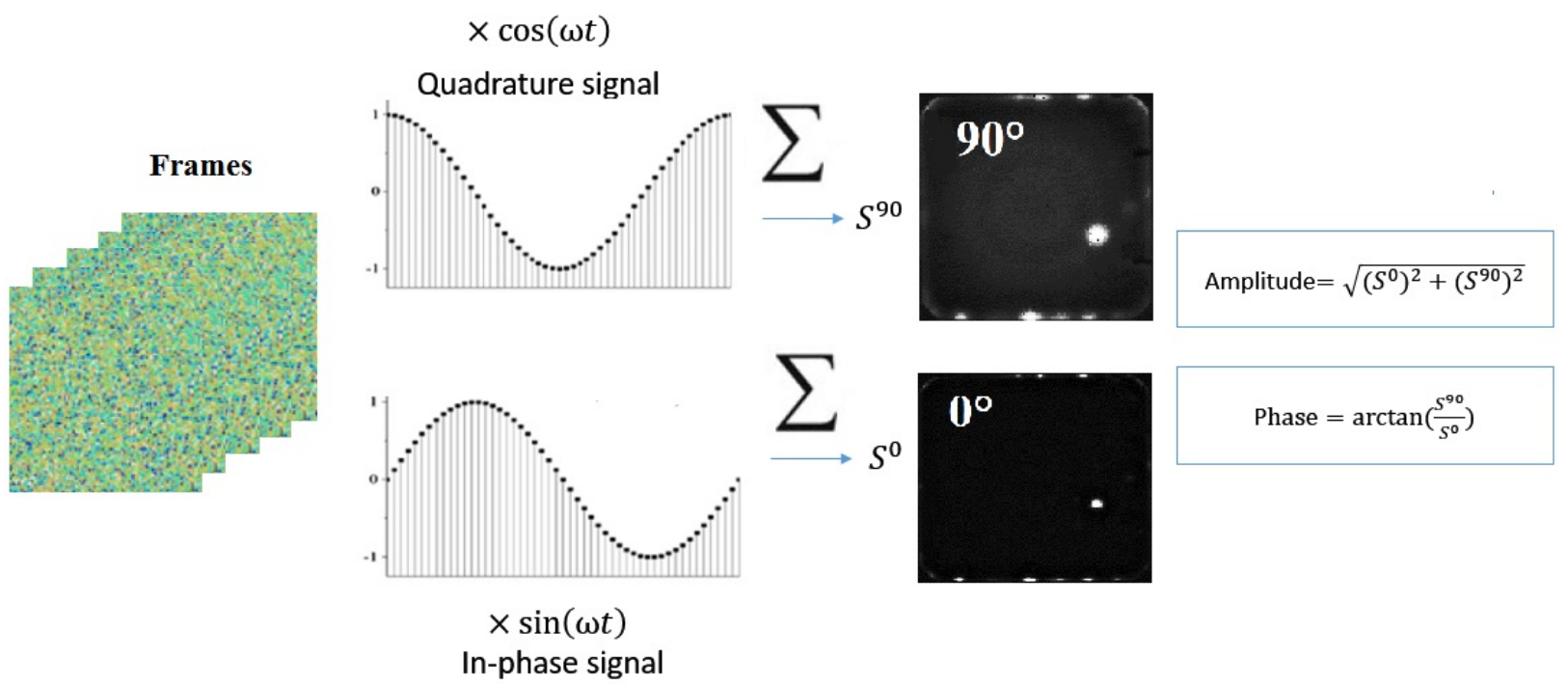

The high frequency (LDR frequency) acts as a carrier delivering heating energy into the specimen and the blind frequency produces the desired thermal wave length.

\subsection{Thermal wave processing by using the lock-in algorithm}

The lock-in procedure is now applied to the thermal waves on the surface of the specimen. The lock-in thermography takes advantage of sine/cosine correlations. The weighting factors of the in-phase and quadrature components of the correlated signals are measured by the sine and cosine functions, respectively [12]. These two components of each surface pixel are then used to generate the phase and amplitude images as shown in Fig. 7. It is important to note that to construct the phase and amplitude images, at least four frames per modulation frequency is required $[12,29,30]$. Therefore, a frame rate of $50 \mathrm{~Hz}$ is selected for the thermal

Figure 7: Schematic of the lock-in process applied to the frames obtained by an IR camera.

Theoretical modulation frequencies are given in Table 4. However, in order to analyze the role of the modulation frequency on the phase and amplitude images, a range of modulation frequencies from $0.009 \mathrm{~Hz}$ to $0.035 \mathrm{~Hz}$, corresponding to $C=1$ to 2 , is considered. To quantify 
the result, a signal-to-noise ratio (SNR) is calculated for each amplitude and phase image by using the following equations [31]:

$$
\begin{aligned}
S N R(A m) & =\frac{\left|\overline{A m_{D}}-\overline{A m_{N}}\right|}{\sigma\left(A m_{N}\right)} \\
S N R(P h) & =\frac{\left|\overline{P h_{D}}-\overline{P h_{N}}\right|}{\sigma\left(P h_{N}\right)}
\end{aligned}
$$

where $\overline{A m_{D}}$ and $\overline{P h_{D}}$ are respectively the mean values of amplitude and phase in a defective region, $\overline{A m_{N}}$ and $\overline{P h_{N}}$ are respectively the mean values of the amplitude and phase in a nondefective region, $\sigma\left(A m_{N}\right)$ and $\sigma\left(P h_{N}\right)$ are respectively the standard deviation of amplitude and phase in a non-defective region, respectively.

The SNR results show that the maximum SNR in amplitude and phase images occurs at $0.009 \mathrm{~Hz}$ and $0.02 \mathrm{~Hz}$, respectively, as shown in Figs. 8 and 9. The value of the parameter $C$, defined by Eq. 14, is given next to each marker used for plotting Figs. 8 and 9. Images obtained by the lock-in algorithm and SNR graphs show that the maximum contrast in amplitude and phase is achieved at modulation frequencies of $0.009 \mathrm{~Hz}$ and $0.02 \mathrm{~Hz}$, respectively.

To get more insight into this analysis, Fig. 10 illustrates a typical example of the phase and amplitude images at the optimal and the extreme limits of the frequency range. Figure 10 shows that the minimum and maximum temperature amplitudes occur at $0.03 \mathrm{~Hz}$ and at $0.009 \mathrm{~Hz}$, respectively, whereas the minimum and maximum phase contrasts are observed at $0.009 \mathrm{~Hz}$ and at $0.02 \mathrm{~Hz}$, respectively.

To further measure the damaged area, the Otsu's thresholding (graythresh) [4, 32] was applied to both the phase and amplitude images obtained by the lock-in algorithm. Fig. 11 shows a typical example of this thresholding process on amplitude and phase images at 0.02 Hz. The relative errors compared to the real value of the defective area (314.16) are then listed in Table 5.

The results confirm that the phase images can estimate the damaged area much better than the amplitude images, see Table 5. Results obtained by combining the lock-in algorithm, SNR analysis and the Otsu's thresholding show that the $0.02 \mathrm{~Hz}$ frequency is the 


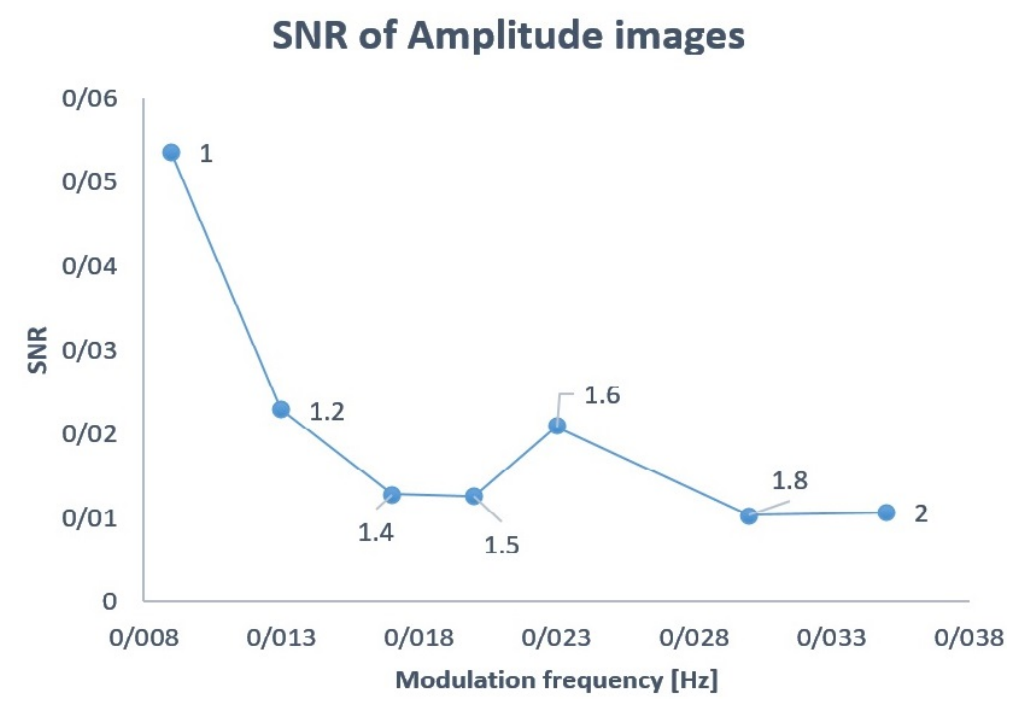

Figure 8: SNR of amplitude images as a function of modulation frequency showing that the maximum SNR occurs at $0.009 \mathrm{~Hz}$.

\section{SNR of Phase images}

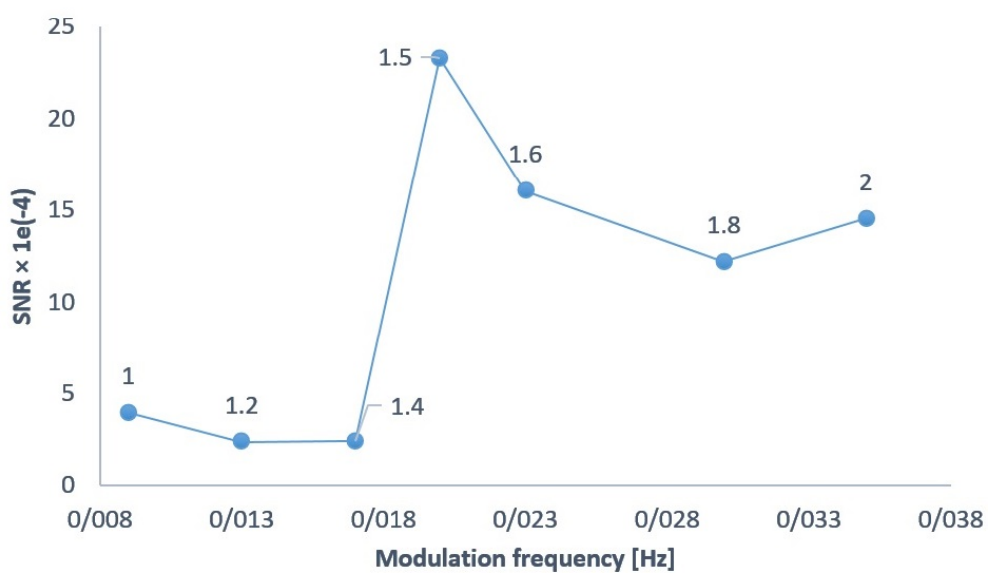

Figure 9: SNR of phase images as a function of modulation frequency showing that the maximum SNR occurs at $0.02 \mathrm{~Hz}$. has also been used as a modulation frequency in the experimental analysis conducted in [15]. 
a)

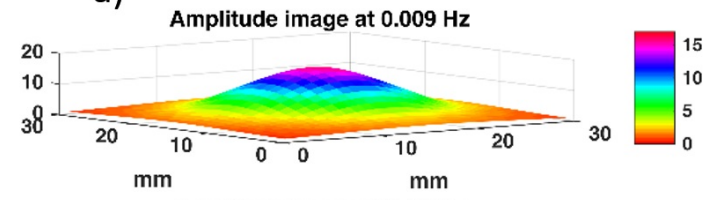

Amplitude image at $0.02 \mathrm{~Hz}$

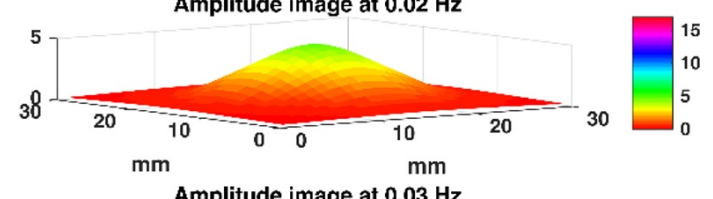

Amplitude image at $0.03 \mathrm{~Hz}$

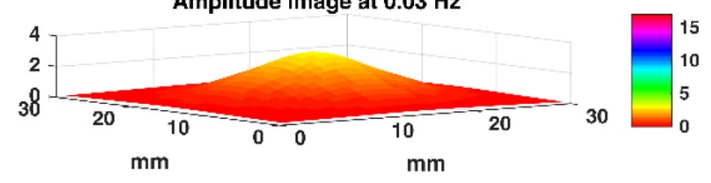

b) Phase image [rad] at $0.009 \mathrm{~Hz}$
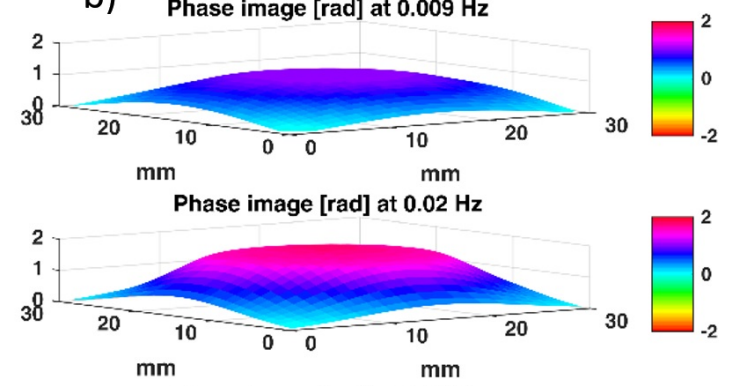

Phase image [rad] at $0.03 \mathrm{~Hz}$

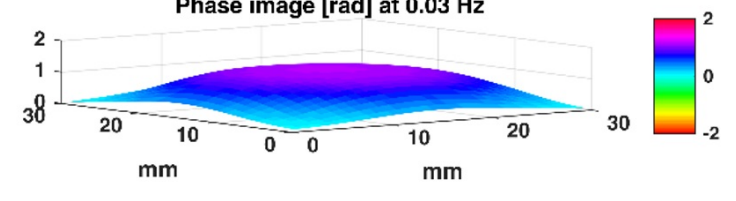

Figure 10: A typical example of amplitude and phase images obtained by the lock-in algorithm at the modulation frequency of $0.009 \mathrm{~Hz}, 0.02 \mathrm{~Hz}$, and $0.03 \mathrm{~Hz}$, a) Amplitude images, b) Phase images.
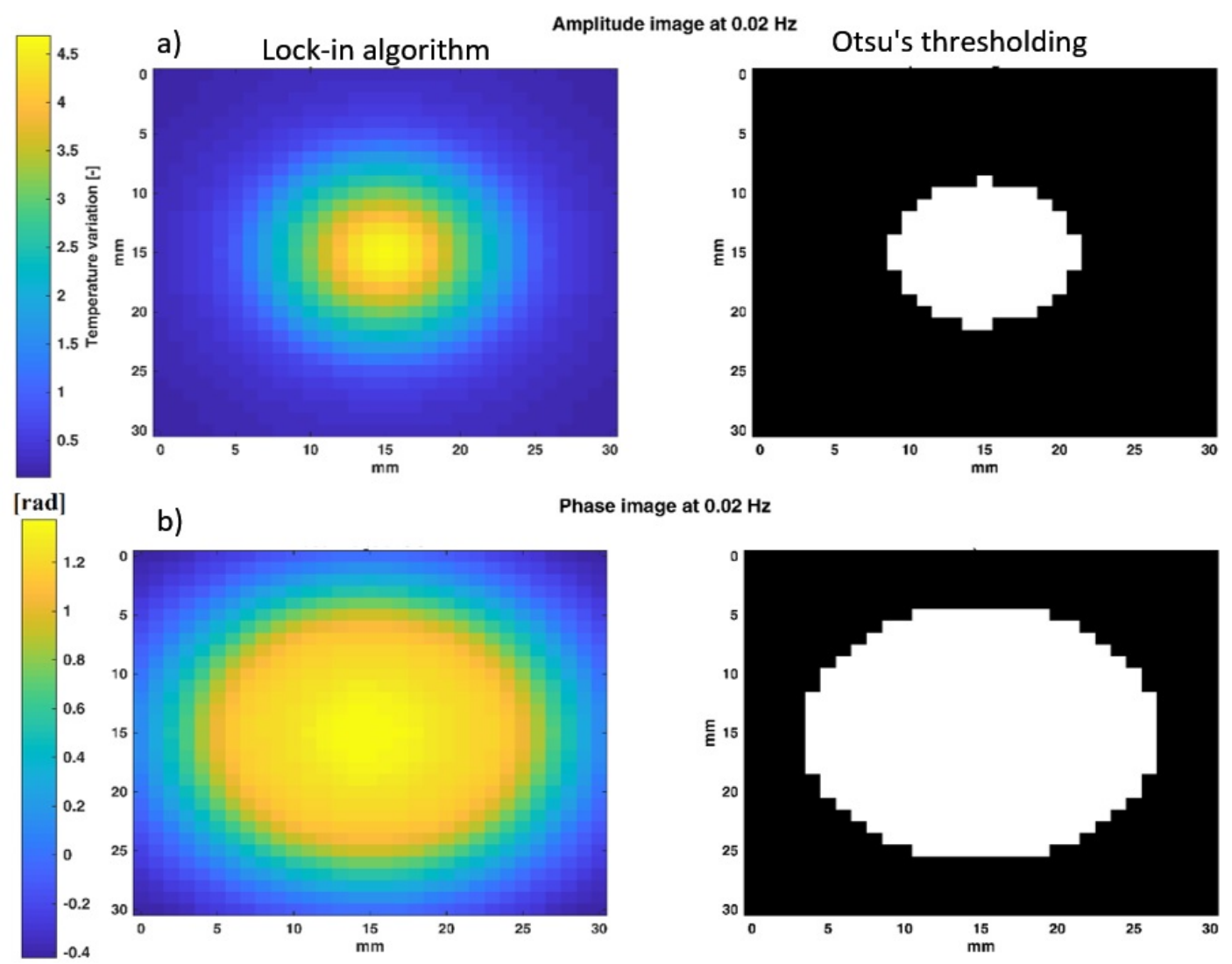

Figure 11: Typical images obtained by lock-in algorithm and Otsu's thresholding at $0.02 \mathrm{~Hz}$, a) Amplitude,

b) Phase, showing that the phase images are more accurate in estimating the size of defect. 
Table 5: Calculated defective area for the FBH defect based on the Otsu's thresholding shown in Fig.11.

\begin{tabular}{ccccc}
\hline $\begin{array}{c}\text { Modulation frequency } \\
{[\mathrm{Hz}]}\end{array}$ & $\begin{array}{c}\text { Amplitude images } \\
\left(\mathrm{mm}^{2}\right)\end{array}$ & $\begin{array}{c}\text { Error } \\
{[\%]}\end{array}$ & $\begin{array}{c}\text { Phase } \\
\text { Images }\left(\mathrm{mm}^{2}\right)\end{array}$ & $\begin{array}{c}\text { Error } \\
{[\%]}\end{array}$ \\
\hline 0.009 & 120.39 & 61.7 & 396.51 & 26.2 \\
0.02 & 108.51 & 65.5 & 359.9 & 14.5 \\
0.03 & 100.28 & 68.1 & 394.91 & 25.7 \\
\hline
\end{tabular}

\subsection{Verification}

In this Section, we will verify the numerical results obtained for the thermal pattern and temperature variations by comparing them with the experimental results reported in the literature $[2,3,15]$.

Figure 12 compares the experimental and simulated results of temperature variations of the specimen as a function of the input voltage. As the driving voltage increases, the vibration amplitude and the elastic strain energy density in the defect (FBH) area also increases. A quadratic dependence between the input voltage and the temperature variation is also observed, see Eqs. (8) and (9).

Moreover, Fig. 13 illustrates the simulated temperature variations of the defect area at $30 \mathrm{~V}$ input voltage with one modulation cycle of $0.02 \mathrm{~Hz}$. The temperature variation of 380 $\mathrm{mK}$ at the time instant of $50 \mathrm{~s}$ is in good agreement with the temperature variations shown in Fig. 12 at $30 \mathrm{~V}$. It is evident that the temperature variations decline towards the initial temperature value when the excitation is stopped at $50 \mathrm{~s}$.

In Fig. 14, the thermal patterns measured on the surface of the specimen around the flat-bottomed hole are plotted by using the FEM and experimental results [2, 3]. It can be observed that the FEM thermosonic images are in good agreement with experimental results reported in [3]. The additional white ring observed in the experimental thermosonic image of the FBH is due to a shallow step formed around the FBH during the manufacturing process [3]. 


\section{Temperature-Input voltage}

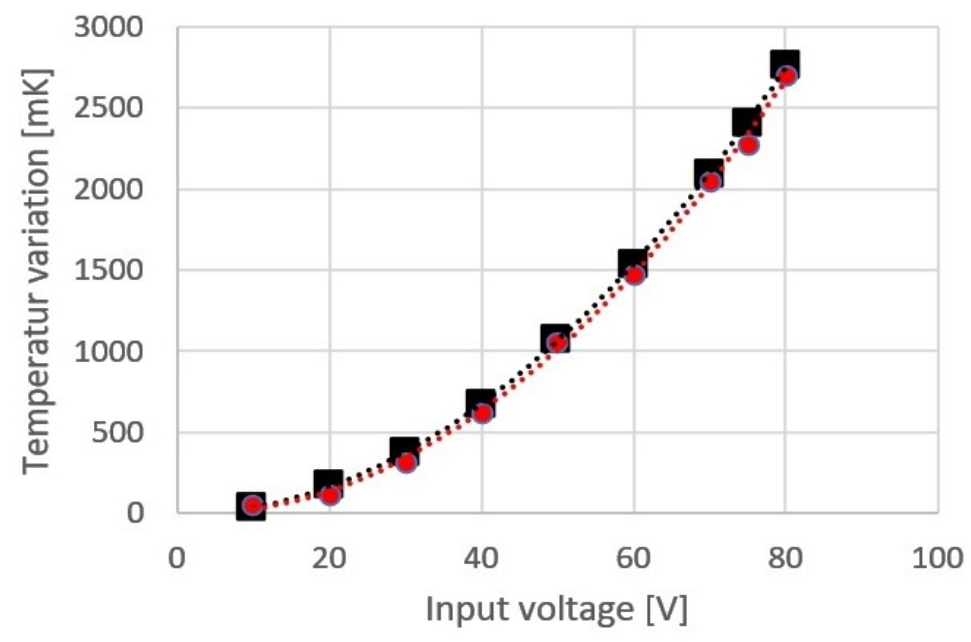

- FEM experiment

Figure 12: A Comparison of temperature variations as a function of input voltage between FEM and experimental data reported in [15] shows good agreement between the numerical and experimental data.

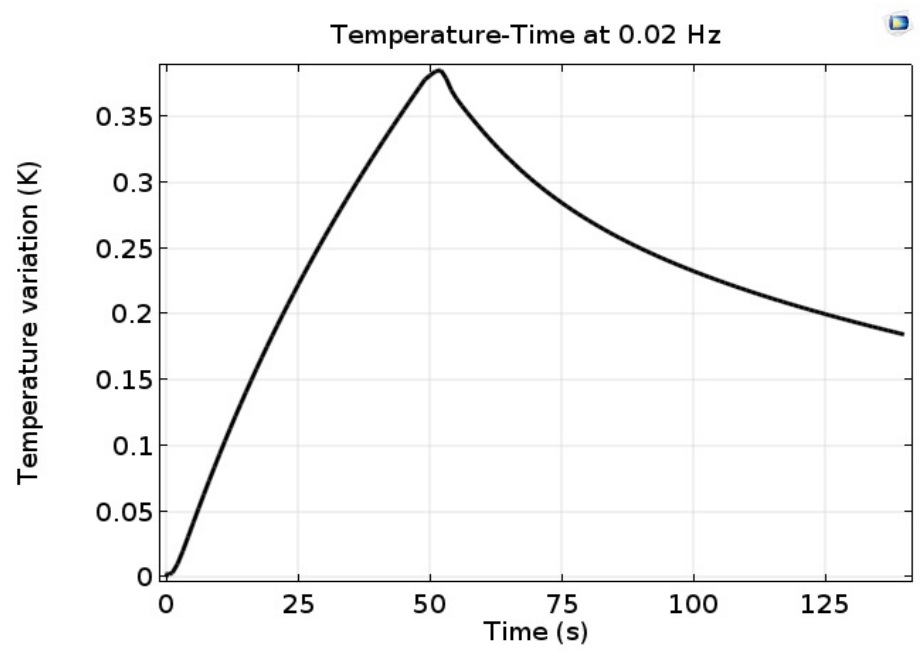

Figure 13: Temperature variation of $\mathrm{FBH}$ at LDR frequency with $30 \mathrm{~V}$ input voltage, showing that the maximum temperature variation during one modulation cycle is about $0.38 \mathrm{~K}$. This is in a good agreement with the temperature variations of Fig.12 at 30V input. 


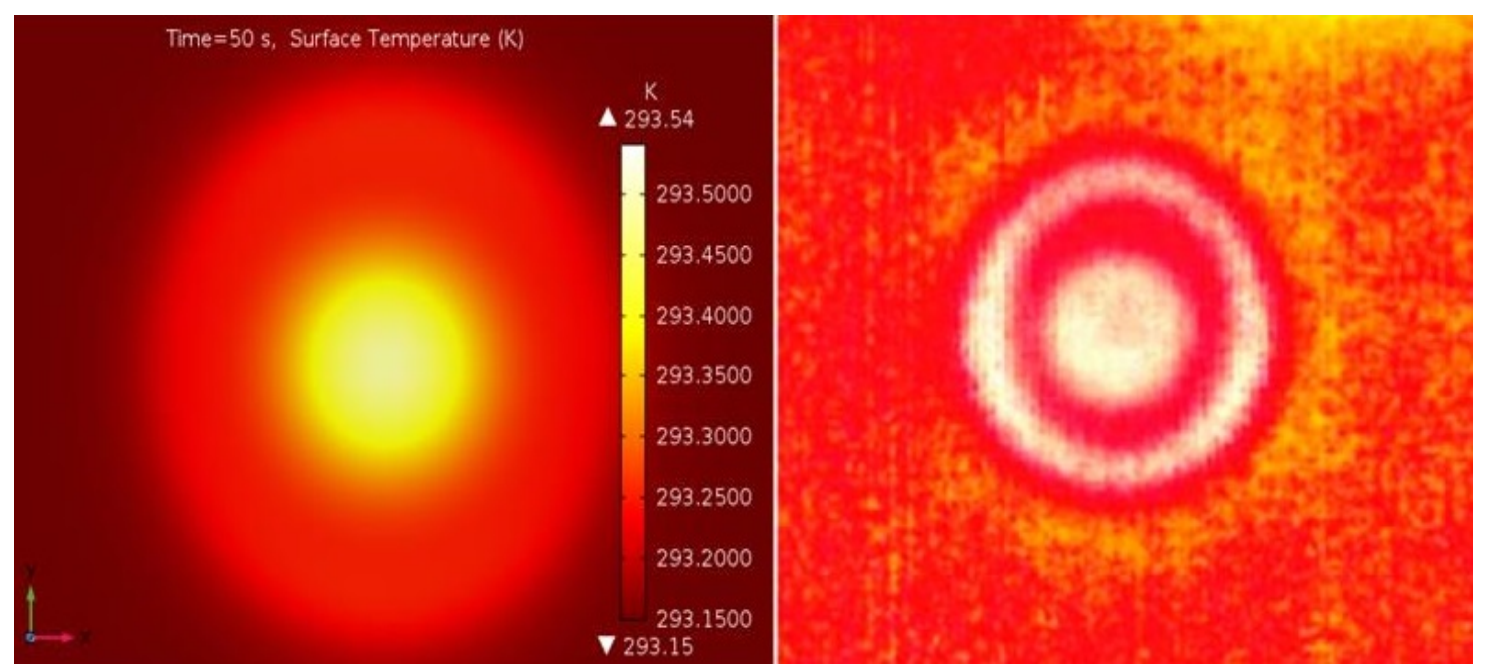

Figure 14: Thermal patterns at the defect area (FBH), a) FEM results, b) experimental results $[2,3]$. Note that an extra ring has emerged in the experimental thermosonic image due to the "stretching" of the FBH defect near to its boundary.

\section{Conclusion}

In this paper, a PMMA plate structure containing a flat-bottomed-hole (FBH) was simulated by finite element method in COMSOL Multiphysics software package. The plate was excited by a chirp signal through a piezo-ceramic actuator mounted on the structure. The out-of-plane components of the top surface displacement signal were then used to measure the local defect resonance (LDR) frequency of the FBH through a principal component analysis algorithm. The LDR frequencies obtained from the theoretical equations, PCA algorithm, and the published experimental results in $[2,15]$ were compared with the calculated LDR through an eigenfrequency analysis. The results confirm that the Principal Component Analysis (PCA) can accurately estimate the LDR frequency.

Thermal diffusion length, which corresponds to the modulation frequency, was found to be an essential parameter in the study of phase and amplitude of thermal waves. To construct the phase and amplitude images, the lock-in algorithm was applied to thermal waves. The thermal pattern at the defect area and the effect of the input voltage on the temperature were validated by comparison with the experimental data available in the literature $[2,3,15]$. 
The results confirmed a quadratic dependence between the driving voltage and temperature variations. Furthermore, the optimal modulation frequency was found by quantifying the amplitude and phase images and estimating the defect size.

Future research is expected to explore the identification and sizing of other defect types such as cracks, porosity and delamination. Moreover, the effect of material properties such as anisotropy and homogeneity as observed in composite and multilayered materials on vibrothermography testing is also interesting topics that should be investigated.

\section{References}

[1] X. Guo, An analytical model and parametric analysis of ultrasound-excited infrared thermography, Quantitative InfraRed Thermography Journal 12 (2015) 137-148.

[2] I. Solodov, M. Rahammer, D. Derusova, G. Busse, Highly-efficient and noncontact vibro-thermography via local defect resonance, Quantitative InfraRed Thermography Journal 12 (2015) 98-111.

[3] I. Solodov, D. Derusova, M. Rahammer, Thermosonic chladni figures for defect-selective imaging, Ultrasonics 60 (2015) 1-5.

[4] J. Hettler, M. Tabatabaeipour, S. Delrue, K. Van Den Abeele, Detection and characterization of local defect resonances arising from delaminations and flat bottom holes, Journal of Nondestructive Evaluation 36 (2017) 2.

[5] T. Zweschper, A. Dillenz, G. Riegert, D. Scherling, G. Busse, Ultrasound excited thermography using frequency modulated elastic waves, Insight-Non-Destructive Testing and Condition Monitoring 45 (2003) 178-182.

[6] S. M. Parvasi, C. Xu, Q. Kong, G. Song, Detection of multiple thin surface cracks using vibrothermography with low-power piezoceramic-based ultrasonic actuatorâĂŤa numerical study with experimental verification, Smart Materials and Structures 25 (2016) 055042.

[7] X. Han, M. Sarwar Islam, G. Newaz, L. Favro, R. Thomas, Finite element modeling of the heating of cracks during sonic infrared imaging, Journal of applied physics 99 (2006) 074905.

[8] R. Montanini, F. Freni, Investigation of heat generation sources in sonic infrared thermography using laser doppler vibrometry, Proceedings of Quantitative Infrared Thermography QIRT (2012).

[9] J. Renshaw, J. C. Chen, S. D. Holland, R. B. Thompson, The sources of heat generation in vibrothermography, NDT \& E International 44 (2011) 736-739.

[10] S. D. Holland, C. Uhl, Z. Ouyang, T. Bantel, M. Li, W. Q. Meeker, J. Lively, L. Brasche, D. Eisenmann, Quantifying the vibrothermographic effect, NDT \& E International 44 (2011) 775-782. 
[11] S. D. Holland, C. Uhl, J. Renshaw, Toward a viable strategy for estimating vibrothermographic probability of detection, in: AIP Conference Proceedings, volume 975, AIP, 2008, pp. 491-497.

[12] O. Breitenstein, W. Warta, M. C. Schubert, M. Langenkamp, Lock-in thermography: Basics and use for evaluating electronic devices and materials, volume 10, Springer, 2018.

[13] A. Gleiter, G. Riegert, T. Zweschper, G. Busse, et al., Ultrasound lock-in thermography for advanced depth resolved defect selective imaging, Insight-Non-Destructive Testing and Condition Monitoring 49 (2007) 272 .

[14] A. Salazar, A. Mendioroz, E. Apiñaniz, A. Oleaga, P. Venegas, I. Sáez-Ocáriz, Characterization of delaminations by lock-in vibrothermography, in: Journal of Physics: Conference Series, volume 214, IOP Publishing, 2010, p. 012079 .

[15] I. Solodov, Local defect resonance (ldr): A route to highly efficient thermosonic and nonlinear ultrasonic ndt, in: AIP Conference Proceedings, volume 1581, AIP, 2014, pp. 1663-1670.

[16] I. Solodov, J. Bai, G. Busse, Resonant ultrasound spectroscopy of defects: case study of flat-bottomed holes, Journal of Applied Physics 113 (2013) 223512.

[17] W. Thomson, M. Dahleh, Theory of vibration with applications, 1993, New Jersey: A Simon \& Schuster Company (1993).

[18] J. P. Holman, Heat transfer (mcgraw-hill series in mechanical engineering), 2010.

[19] X. Guo, L. Zhu, 14th quantitative infrared thermography conference, volume 47, Germany, 2018, pp. $178-184$.

[20] J. K. Miller, D. C. Woods, J. F. Rhoads, Thermal and mechanical response of particulate composite plates under inertial excitation, Journal of Applied Physics 116 (2014) 244904.

[21] www.guiguw.com

[22] P. Fellinger, R. Marklein, K. Langenberg, S. Klaholz, Numerical modeling of elastic wave propagation

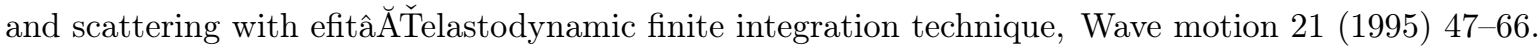

[23] C. A. Leckey, K. R. Wheeler, V. N. Hafiychuk, H. Hafiychuk, D. A. Timuçin, Simulation of guidedwave ultrasound propagation in composite laminates: Benchmark comparisons of numerical codes and experiment, Ultrasonics 84 (2018) 187-200.

[24] www.produktinfo.conrad.com

[25] A. Ilin, T. Raiko, Practical approaches to principal component analysis in the presence of missing values, Journal of Machine Learning Research 11 (2010) 1957-2000.

[26] I. T. Jolliffe, J. Cadima, Principal component analysis: a review and recent developments, Philosophical Transactions of the Royal Society A: Mathematical, Physical and Engineering Sciences 374 (2016) 20150202.

[27] C. Ibarra Castanedo, Quantitative subsurface defect evaluation by pulsed phase thermography: depth 
retrieval with the phase (2005).

[28] S. Ranjit, K. Kang, W. Kim, Investigation of lock-in infrared thermography for evaluation of subsurface defects size and depth, International Journal of Precision Engineering and Manufacturing 16 (2015) $2255-2264$.

[29] O. Breitenstein, J. Rakotoniaina, F. Altmann, T. Riediger, M. Gradhand, New developments in ir lock-in thermography, in: Proc. 30th ISTFA, 2004, pp. 595-599.

[30] N. Tabatabaei, Development of Frequency and Phase Modulated Thermal-wave Methodologies for Materials Non-destructive Evaluation and Thermophotonic Imaging of Turbid Media, Ph.D. thesis, 2012.

[31] J. Liu, W. Yang, J. Dai, Research on thermal wave processing of lock-in thermography based on analyzing image sequences for ndt, Infrared Physics \& Technology 53 (2010) 348-357.

[32] N. Otsu, A threshold selection method from gray-level histograms, IEEE transactions on systems, man, and cybernetics 9 (1979) 62-66. 